\title{
Comprehensive genomic profiling of orbital and ocular adnexal lymphomas identifies frequent alterations in MYD88 and chromatin modifiers: new routes to targeted therapies
}

Andi K Cani ${ }^{1,2}$, Moaaz Soliman ${ }^{3}$, Daniel H Hovelson ${ }^{1,4}$, Chia-Jen Liu ${ }^{1,2}$, Andrew S McDaniel ${ }^{1,2}$, Michaela J Haller ${ }^{1,2}$, Jarred V Bratley ${ }^{1,2}$, Samantha E Rahrig ${ }^{1,2}$, Qiang $\mathrm{Li}^{3}$, César A Briceño ${ }^{3}$, Scott A Tomlins ${ }^{1,2,5,6}$ and Rajesh C Rao ${ }^{2,3,6,7}$

${ }^{1}$ Michigan Center for Translational Pathology, University of Michigan Medical School, Ann Arbor, MI, USA; ${ }^{2}$ Department of Pathology, University of Michigan Medical School, Ann Arbor, MI, USA; ${ }^{3}$ Department of Ophthalmology and Visual Sciences, W.K. Kellogg Eye Center, University of Michigan Medical School, Ann Arbor, MI, USA; ${ }^{4}$ Department of Computational Medicine and Bioinformatics, University of Michigan Medical School, Ann Arbor, MI, USA; ${ }^{5}$ Department of Urology, University of Michigan Medical School, Ann Arbor, MI, USA; ${ }^{6}$ Comprehensive Cancer Center, University of Michigan Medical School, Ann Arbor, MI, USA and ${ }^{7}$ Section of Ophthalmology, Surgical Service, Veterans Administration Ann Arbor Healthcare System, Ann Arbor, MI, USA

Non-Hodgkin lymphoma of the orbit and ocular adnexa is the most common primary orbital malignancy. Treatments for low- (extra-nodal marginal zone and follicular lymphomas) and high-grade (diffuse large B-cell lymphoma) are associated with local and vision-threatening toxicities. High-grade lymphomas relapse frequently and exhibit poor survival rates. Despite advances in genomic profiling and precision medicine, orbital and ocular adnexal lymphomas remain poorly characterized molecularly. We performed targeted next-generation sequencing (NGS) profiling of 38 formalin-fixed, paraffin-embedded orbital and ocular adnexal lymphomas obtained from a single-center using a panel targeting near-term, clinically relevant genes. Potentially actionable mutations and copy number alterations were prioritized based on gain- and loss-of-function analyses, and catalogued, approved, and investigational therapies. Of 36 informative samples, including marginal zone lymphomas $(n=20)$, follicular lymphomas $(n=9)$, and diffuse large B-cell lymphomas $(n=7), 53 \%$ harbored a prioritized alteration (median =1, range $0-5 /$ sample). MYD88 was the most frequently altered gene in our cohort, with potentially clinically relevant hotspot gain-of-function mutations identified in $71 \%$ of diffuse large B-cell lymphomas and $25 \%$ of marginal zone lymphomas. Prioritized alterations in epigenetic modulators were common and included gain-of-function EZH2 and loss-of-function ARID1A mutations (14\% of diffuse large B-cell lymphomas and $22 \%$ of follicular lymphomas contained alterations in each of these two genes). Single prioritized alterations were also identified in the histone methyltransferases KMT2B (follicular lymphoma) and KMT3B (diffuse large B-cell lymphoma). Loss-of-function mutations and copy number alterations in the tumor suppressors TP53 (diffuse large B-cell and follicular lymphoma), CDKN2A (diffuse large B-cell and marginal zone lymphoma), PTEN (diffuse large B-cell lymphoma), ATM (diffuse large B-cell lymphoma), and NF1 (diffuse large B-cell lymphoma), and gainof-function mutations in the oncogenes HRAS (follicular lymphoma) and NRAS (diffuse large B-cell lymphoma) were also observed. Together, our study demonstrates that NGS can be used to profile routine formalin-fixed, paraffin-embedded orbital and ocular adnexal lymphomas for identification of somatic-driving alterations and nomination of potential therapeutic strategies.

Modern Pathology (2016) 29, 685-697; doi:10.1038/modpathol.2016.79; published online 22 April 2016

Correspondence: Dr SA Tomlins, MD, PhD, University of Michigan Medical School, 1524 BSRB, 109 Zina Pitcher Place, Ann Arbor, MI 48109, USA or Dr RC Rao, MD, Department of Pathology, Department of Ophthalmology \& Visual Sciences, W.K. Kellogg Eye Center, University of Michigan Medical School, 1000 Wall Street, Brehm Room 8333, Ann Arbor, MI 48105, USA.

E-mail: tomlinss@umich.edu or rajeshr@umich.edu

Received 25 December 2015; revised 21 March 2016; accepted 24 March 2016; published online 22 April 2016 


\section{Introduction}

Non-Hodgkin lymphomas (NHLs) of the orbital and ocular adnexa represent $10-15 \%$ of all tumors present in the orbit, eyelids, conjunctiva, and lacrimal apparatus (ie, the 'adnexa') and are the most common primary orbital cancer in adults. ${ }^{1,2}$ Alarmingly, orbital and ocular adnexal lymphomas have shown an increasing incidence in some populations $(\sim 6 \%$ yearly increase in white Americans from an analysis of Surveillance, Epidemiology, and End Results data). ${ }^{3}$

Orbital and ocular adnexal lymphomas are B-cell proliferations that include low-grade tumors such as extra-nodal marginal zone (also known as mucosaassociated lymphoid tissue lymphoma (MALT)) and follicular lymphoma, as well as high-grade tumors such as mantle cell lymphoma and diffuse large B-cell lymphomas. These subtypes account for $\sim 50 \%, 10-20 \%, 5-10 \%$, and $5-10 \%$ of orbital and ocular adnexal lymphomas, respectively., ${ }^{4,5}$

The molecular etiology of orbital and ocular adnexal lymphoma heterogeneous subtypes is diverse and not well understood. MZLs are frequently associated with translocations: API2MALT1, IGH-MALT1, IGH-BCL10, and IGH-FOXP1, as well as trisomy of chromosome 3 and $18 .{ }^{6}$ Such aberrations activate pathways that converge on the nuclear factor- $\kappa \mathrm{B}$ (NF- $\kappa \mathrm{B})$ complex, leading to orbital and ocular adnexal lymphomagenesis. ${ }^{6}$ Molecular studies of orbital and ocular adnexal follicular and diffuse large B-cell lymphomas are few, owing to the rarity of primary tumors of these subtypes in these locations. Thus, molecular alterations in these subtypes are presumed to follow systemic FL and DLBCL pathogenetic patterns. In systemic diffuse large B-cell lymphomas and BCL2/IGH and $M Y C$ rearrangements, lesions associated with $\mathrm{NF}-\kappa \mathrm{B}$ activation (TNFAIP3, CARD11, CD79B, and MYD88) and epigenetic alterations (CREBBP, EP300, and $E Z H 2)$ have been described, but there are also many altered genes that encode for structural (eg, $P C L O$ ) and signaling proteins (P2RY8 and TNFRSF14). ${ }^{7,8}$ Systemic follicular lymphomas share some lesions with diffuse large B-cell lymphomas, including $I G H$ and BCL2 rearrangements ${ }^{9,10}$ and alterations in EZH2 and TNFRSF14, ${ }^{11}$ but also exhibits distinct changes including somatic mutations that lead to loss of function of EPHA2 ephrin receptor ${ }^{12}$ and acquisition of $\mathrm{N}$-glycosylation motifs in immunoglobulin genes. ${ }^{13}$

Although the mainstay of treatment for most primary orbital and ocular adnexal lymphomas (low grade), external beam radiation therapy, results in high rates of local control, the rate of distant relapse can reach $40 \%{ }^{14,15}$ and local toxicities such as keratitis, dry eye, cataract, neovascular glaucoma, optic nerve neuropathy, and retinopathy can lead to vision loss or blindness. ${ }^{16,17}$ Survival rates vary with orbital and ocular adnexal lymphoma subtype. In general, marginal zone and most follicular lymphomas (low-grade tumors) of the orbital and ocular adnexal region are treated with external beam radiation therapy and 10-year disease-specific survival rates, depending on stage, range from 94 to $98 \% .{ }^{18,19}$ In contrast, diffuse large B-cell lymphomas and some follicular lymphomas of the orbital and ocular adnexal region are tumors that often lead to, or are a manifestation of, disseminated systemic disease and are treated with a combination of external beam radiation therapy, surgery, and/or systemic chemotherapy. These diffuse large B-cell lymphomas relapse frequently and have 10-year disease-specific survival rates ranging from 18 to $55 \% .^{20}$

In summary, the genomic drivers of orbital and ocular adnexal lymphomas, especially those that underlie orbital and ocular adnexal follicular and diffuse large B-cell lymphomagenesis remain poorly understood, due, in part, to the rarity of primary tumors at these locations. Although lowgrade tumors such as marginal zone and follicular lymphomas are frequently curable by external beam radiation therapy, they are often associated with vision-threatening local toxicities and can relapse at distant locations. Treatments such as external beam radiation therapy, surgery and systemic chemotherapy for high-grade orbital and ocular adnexal lymphomas are associated with vision threatening and systemic toxicities. Moreover, diffuse large B-cell lymphomas frequently relapse and are often deadly. Taken together, these data demonstrate an unmet need to develop novel treatment strategies for both low- and high-grade orbital and ocular adnexal lymphomas.

The development of novel therapies for orbital and ocular adnexal lymphomas has been hampered by the limited nature of studies exploring genomic drivers of the disease. Although cytogenetic, copy number and transcript, and immunohistochemistrybased profiling studies have been reported, ${ }^{21-25}$ these represent limited cohorts mainly assessing marginal zone lymphoma, the orbital and ocular adnexal lymphoma subtype most frequently associated with good prognosis. Importantly, although next-generation sequencing (NGS) studies have defined genetic alterations and potential therapeutic targets in non-orbital lymphomas, such approaches have not been applied to orbital and ocular adnexal lymphomas. Hence, in order to define potentially actionable somatic mutations and copy number alterations in orbital and ocular adnexal lymphomas, we performed NGS on 38 formalin-fixed, paraffin embedded orbital and ocular adnexal lymphomas, including marginal zone, follicular and diffuse large B-cell histologic subtypes. To do this, we subjected our set of orbital and ocular adnexal lymphomas to a NGS-based panel enriched for alterations in solid tumors and lymphomas, a modified version of which is being used in the National Cancer Institute-Molecular Analysis for Therapy Choice (NCI-MATCH) Trial, ${ }^{26}$ a new trial that has matched 
patients with advanced lymphomas and solid tumors, which have not responded (or never responded), to approved or investigational therapeutics based on their prioritized mutation profile rather than the site of tumor origin. ${ }^{27}$

\section{Materials and methods}

\section{Case Selection}

The study was carried out at the highest ethical standards and with the approval of the University of Michigan Institutional Review Board. We identified a cohort of 38 archived, routine clinical formalin-fixed, paraffin-embedded orbital lymphoma specimens from the University of Michigan Department of Pathology Tissue Archive for NGS. Clinicopathological information for each case was obtained from the clinical archive. Hematoxylin and eosin (H\&E)-stained slides and immunostains (where available) were reviewed for all cases by board-certified Anatomic Pathologists (ASM and SAT), to ensure sufficient tumor content (70-90\%).

\section{Targeted NGS}

Targeted NGS was performed essentially as previously described. ${ }^{26,28,29}$ For each specimen, $3-7 \times 10 \mu \mathrm{m}$ formalin-fixed, paraffin-embedded sections were cut from a single representative block per case, using macrodissection with a scalpel as needed, to enrich for tumor content. DNA was isolated using the Qiagen Allprep formalin-fixed, paraffin-embedded DNA/RNA kit (Qiagen, Valencia, CA) and quantified as previously described. Targeted, multiplexed PCR-based NGS was performed by Ion Torrent NGS using the DNA component of a beta version of the Oncomine Comprehensive Assay, a custom panel comprising 3435 amplicons targeting 126 genes. Genes included in this panel were selected based on pan-solid tumor NGS and copy number profiling data analysis that prioritized somatic, recurrently altered oncogenes, tumors suppressors, genes present in high-level copy gains/losses, and known/investigational therapeutic targets. ${ }^{26}$ Library preparation with barcode incorporation, template preparation, and sequencing using the Ion Torrent Proton sequencer were performed according to the manufacturer's instructions. Data analysis was performed using Torrent Suite 4.0.2, with alignment by TMAP using default parameters, and variant calling using the Torrent Variant Caller plugin (version 4.0-r76860) using default low-stringency somatic variant settings. Variant annotation filtering and prioritization was performed essentially as described, using validated in-house pipelines. ${ }^{26,28-31}$ Briefly, called variants were filtered to remove synonymous or non-coding variants, those with flow-corrected read depths (FDP) $<20$, flow-corrected variant allele containing reads (FAO)
$<6$, variant allele frequencies $(\mathrm{FAO} / \mathrm{FDP})<0.10$, extreme skewing of forward/reverse flow-corrected reads calling the variant $(<0.2$ or $>5)$, or indels within homopolymer runs $>4$. Called variants were filtered using a panel-specific, in-house blacklist. Variants with allele frequencies $>0.5 \%$ in Exome Sequencing Project 6500 (ESP6500) or the 1000 Genomes project, and those reported in ESP6500 or 1000 genomes with observed variant fractions between 0.40 and 0.60 or $>0.9$ were considered germline variants unless occurring at a known hotspot variant. Variants located at the last mapped base (or outside) of amplicon target regions, variants with the majority of supporting reads harboring additional mismatches or indels (likely sequencing error), those in repeat-rich regions (likely mapping artifacts), and variants occurring exclusively in one amplicon if overlapping amplicons cover the variant were excluded. High-confidence somatic variants passing the above criteria were then visually confirmed in Integrative Genomics Viewer (https://www.broadin stitute.org/igv/). We have previously confirmed that these filtering criteria identify prioritized high-confidence somatic variants that pass Sanger sequencing validation with $>95 \%$ accuracy. ${ }^{28-32}$ Copy number analysis from total amplicon read counts provided by the Coverage Analysis Plug-in (v4.0-r77897) was performed essentially as described using a validated approach. ${ }^{26,28,29,33}$ Genes with a $\log _{2}$ copy number estimate of $<-1$ or $>0.6$ were considered to have high-level loss or gain, respectively.

To prioritize potential driving alterations, we used Oncomine software tools (powertools.oncomine. com) to annotate called variants, which use pancancer NGS data to identify genes as oncogenes or tumor suppressors, based on overrepresentation of hotspot or deleterious mutations, respectively. Variants in oncogenes are then considered gainof-function if at a hotspot and variants in tumor suppressors are considered loss-of-function if deleterious or at a hotspot. ${ }^{26,28,30}$ Likewise, high-level copy number alterations were prioritized if they were concordant with the minimal common region analysis used to design the Oncomine Comprehensive Assay (eg, high-level copy number gain in a gene prioritized as amplified/deleted by minimal common region analysis.

\section{Immunohistochemistry}

Immunohistochemistry was performed on the DAKO Autostainer (DAKO, Carpinteria, CA) using DAKO Envision+ and diaminobenzadine as the chromogen. Sections of de-paraffinized Orbital and ocular adnexal lymphomas were labeled with ARID1A (mouse monoclonal, clone PSG3, 1:250, Santa Cruz Biotechnology, Dallas TX, sc-32761), EZH2 (mouse monoclonal, clone 11, 1:100, BD Biosciences, San Jose, CA, 612666), or KMT3B/NSD1 (rabbit polyclonal, 1:250, EMD Millipore, Billerica, MA, 
ABE1009) for $60 \mathrm{~min}$ at ambient temperature. Microwave epitope retrieval in $10 \mathrm{mM}$ Tris/HCl $\mathrm{pH} 9$ containing $1 \mathrm{mM}$ EDTA was used before staining. Appropriate negative (no primary antibody) and positive controls were stained in parallel with each set of tumors studied. Immunostained slides were examined by light microscopy by two pathologists (A.S.M. and S.A.T.). Only staining of the nucleus was marked as a positive expression. The staining was scored semi-quantitatively and recorded based on percent nuclei staining $(0=$ negative, $1=1-25 \%$ immunoreactive cells, $2=26-50 \%$ immunoreactive cells, $3=51-75 \%$ immunoreactive cells, and $4=76-$ $100 \%$ immunoreactive cells) and intensity of staining ( 0 = negative, $1=$ weak, $2=$ moderate, and $3=$ strong). Corresponding sections were also H\&E stained.

\section{Statistics}

Comparisons of the number of mutations and copy number alterations per sample by lymphoma subtype were performed using the Kruskal-Wallis test with post-hoc pairwise comparison of subgroups using R 3.1.0 (R Foundation for Statistical Computing, Vienna, Austria).

\section{Results}

We performed targeted NGS on a cohort of 38 formalin-fixed, paraffin-embedded orbital and ocular adnexal lymphomas comprising 8 diffuse large B-cell, 9 follicular, and 21 marginal zone lymphomas; representative photomicrographs are shown in Figure $1 \mathrm{a}$ and $\mathrm{b}$ and clinical characteristics of all informative patients (see below) are presented in Figure 1c. We isolated an average of $5.5 \mu \mathrm{g}$ DNA per case (range $0.1-24.1 \mu \mathrm{g}$ ) and all samples had $>70 \%$ estimated tumor content by $\mathrm{H} \& \mathrm{E}$ evaluation after macrodissection (as needed). NGS was performed using the DNA component of a beta version of the Oncomine Comprehensive Assay (version 1), a custom panel comprising 3435 amplicons targeting 126 genes, and Ion Torrent-based sequencing on the Proton machine, a modified version of which is being used in the NCI-MATCH trial. ${ }^{27}$ Targeted genes were selected based on pan-solid tumor NGS and copy number profiling data analysis, to prioritize somatic, recurrently altered oncogenes, tumors suppressors, and genes present in high-level copy number alteration, filtered by available or investigational therapeutic targets. ${ }^{26}$

Of the 38 samples assayed, one marginal zone lymphoma (IE-26, primary to orbit/ocular adnexa, Ann Arbor stage IIAE, with no previous treatment) and one diffuse large B-cell lymphoma (IE-31, nonprimary to orbit/ocular adnexa, Ann Arbor stage IAE, with previous chemotherapy and EBRT) sample yielded low-quality sequencing data due to poor genomic DNA quality and were excluded from all subsequent analyses (Figure 1c). Most of the informative 36 lymphomas were primary to the orbit or ocular adnexa, not previously treated, and of early stage. Twenty-seven of 36 tumors were primary to the orbit or ocular adnexa, including 6 of 7 diffuse large B-cell lymphomas, 4 of 9 follicular lymphomas, and 17 of 20 marginal zone lymphomas. Most were not previously treated: none of the diffuse large B-cell lymphomas, 5 of the follicular lymphomas, and 18 of the marginal zone lymphomas. Most of the marginal zone lymphomas ${ }^{15}$ and diffuse large B-cell lymphomas $^{6}$ were Ann Arbor stage IAE, whereas most of the follicular lymphomas were $\mathrm{IAE}^{2}$ or IIAE (Figure 1c). Primary follicular and diffuse large B-cell lymphomas to the orbit and ocular adnexa have rarely been profiled.

Using the Hans algorithm for germinal center B-cell phenotyping, we found that six of the eight diffuse large B-cell lymphomas were of the activated B-cell like phenotype and the remaining two were of the germinal center B-cell phenotype. ${ }^{34}$ One of the germinal center B-cell-positive cases was unable to be sequenced due to poor DNA quality (IE-31).

Across the 36 informative samples, NGS generated an average of 1871816 mapped reads yielding $545 \times$ targeted base coverage (Supplementary Table S1). We identified a total of 41 high-confidence, prioritized somatic alterations (median $n=1$, range $n=0-$ 5 per sample) comprising nonsynonymous point mutations $(n=33)$, short insertions/deletions (indels; $n=2)$, and copy number variations $(n=6)$. All clinical data (including staging, primary site, and previous treatment), prioritized somatic mutations, and high-level copy number alterations for each case are shown in an integrative heat map (Figure 1c) and given in Supplementary Table S2. Prioritized alterations were present in 19 of 36 samples (53\%). Diffuse large B-cell lymphomas harbored the majority of total prioritized alterations (6 of 7 (86\%) with prioritized alterations; median prioritized alterations $n=3$, range $n=0-5)$. Prioritized alterations were identified in 6 of $9(67 \%)$ follicular lymphomas (median prioritized alterations $n=1$, range $n=0-3$ ) and 7 of $20(35 \%)$ marginal zone lymphomas (median prioritized alterations $n=0$, range $n=0-2$ ). The number of prioritized alterations was significantly different between the lymphoma subtypes (Kruskal-Wallis test, $P=0.0014$ ), as shown in Figure 1d.

Across the 36 orbital and ocular adnexal lymphomas, $10(28 \%)$ harbored prioritized gain-of-function mutations in the Toll/interleukin-1 receptor (TIR) domain of myeloid differentiation factor 88 (MYD88), making it the most frequently altered gene (by prioritized alterations) in our cohort. MYD88 is an adaptor protein that binds to the intracellular domains of Toll-like receptors (TLRs) and interleukin 1 receptor on $B$ cells and macrophages, which stimulates the $\mathrm{NF}-\kappa \mathrm{B}$ signaling pathway, and is involved in innate immunity. ${ }^{35}$ The highest proportion of MYD88 hotspot gain-of-function mutations was present in diffuse large B-cell lymphomas 

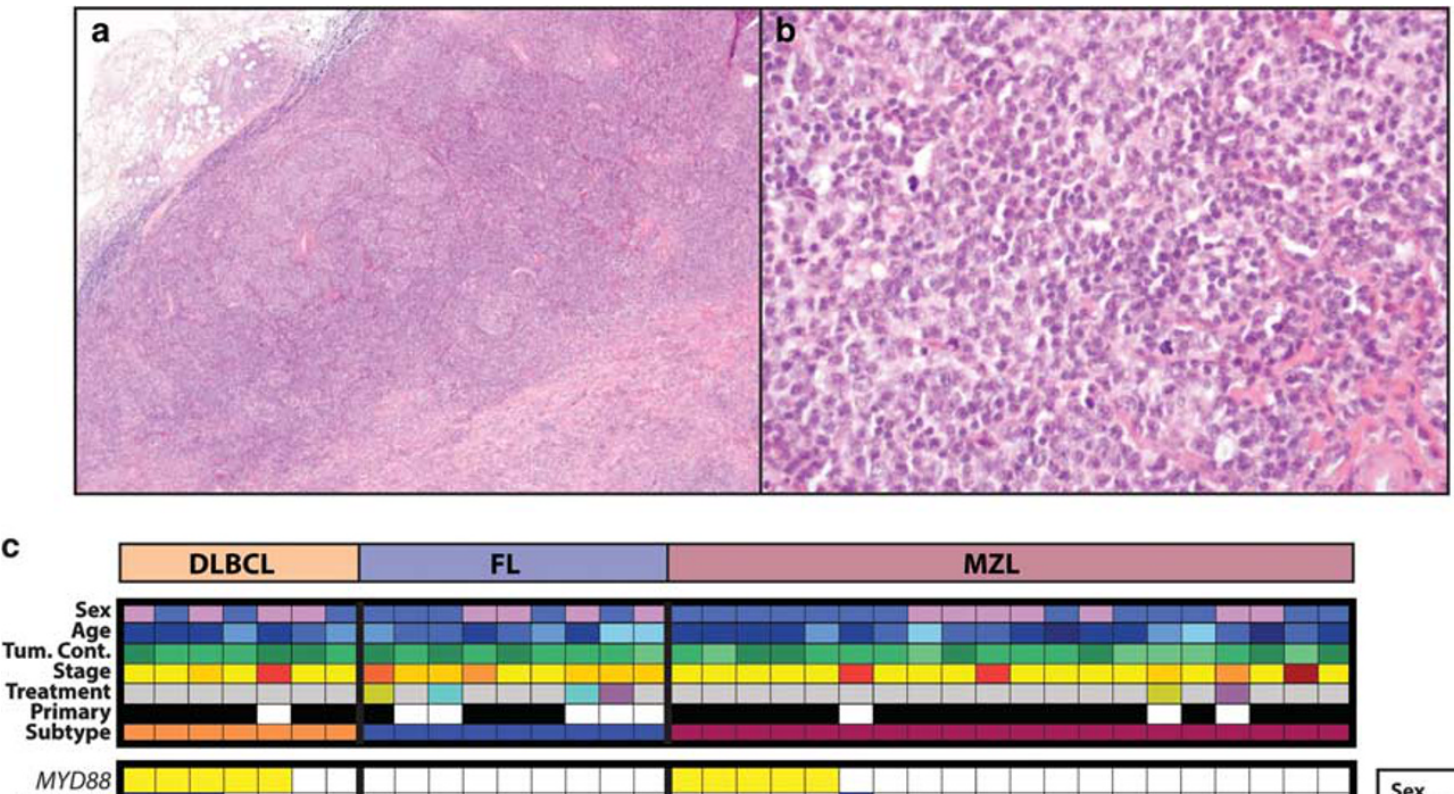

CDKN2A

ARID1A

EZH

FAT1

PTEN

SPEN

ATM

GATA

HRAS

KMT2B

MAP3K1

MED12

NF1

NOTCH1

NRAS

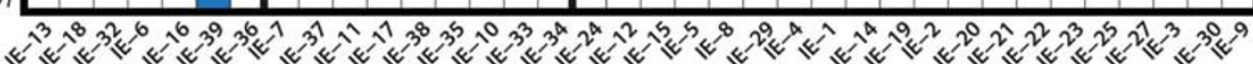

\section{Sex Male \\ - Female}

Age

난 $<5$

$\begin{array}{r}55-65 \\ -65-75 \\ \hline\end{array}$

$65-75$
$75-85$

- $>85$

Tum. Cont.

믈 $70-80 \%$

- >90\%

Stage

$\square$ IAE

- IIAE

- IIIAE

- IIIAES

- IVAE
- IVAES

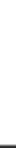

d

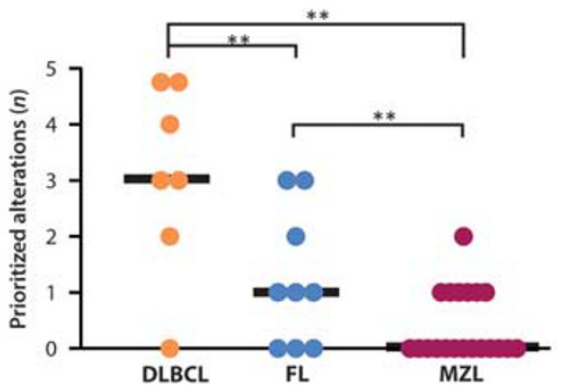

e

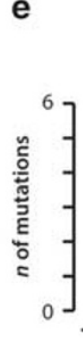

Tumor Suppressors

Treat. Type EBRT - EBRT+Chemo No Prior Tx

Primary

- Yes

Subtype 1. DLBCL - FL 
(5/7, 71\%), including three p.L265P, one p.M232T and one p.S219C mutations. Notably, of the five MYD88 mutation-positive diffuse large B-cell lymphomas, all were of the activated B-cell-like phenotype. The former represents the most frequently altered MYD88 hotspot across human cancers, whereas the latter two mutations occurred at minor hotspots. The remaining MYD88 (TIR domain) mutations occurred in 5 of 20 (25\%) marginal zone lymphoma samples. Of note, MYD88 mutations comprise the majority of total prioritized alterations (5 of $8,63 \%$ ) in our marginal zone lymphoma samples, with three p.L265P mutations and two other TIR domain hotspot mutations (one each of p. M232T and p.I220T). All gain-of-function MYD88 mutations across the cohort are shown in Figure 1e. Taken together, our data demonstrate that MYD88 TIR domain mutations are common in the diffuse large B-cell and marginal zone lymphoma subtypes of orbital and ocular adnexal lymphomas.

Recurrent somatic alterations in histone/chromatin remodeling proteins are frequent in extranodal NHLs outside of the orbit and ocular adnexa. Specifically, 22\% of diffuse large B-cell lymphomas and $7-22 \%$ of follicular lymphomas outside of the orbit and ocular adnexa carry gain-of-function missense mutations (at the p.Y646 hotspot) in the methyltransferase SET domain of the histone H3 lysine 27 trimethylase enhancer of zeste homolog 2 (EZH2). ${ }^{36-38}$ Herein, we identified two of nine follicular lymphomas (22\%) and one of seven diffuse large B-cell lymphomas (14\%) with EZH2 p.Y646 mutations (Figure 1c). The single EZH2 mutant diffuse large B-cell lymphoma case in our cohort was of the germinal center B-cell phenotype. We also identified loss-of-function mutations in tumor suppressor AT-rich interactive domain-containing protein 1A (ARID1A), an epigenetic regulator that is an ATPase-dependent SWI/SNF nucleosome remodeling complex subunit. We identified a prioritized ARID1A nonsense mutation (p.Q1363X) in one of seven (14\%) diffuse large B-cell lymphomas; two of nine $(22 \%)$ follicular lymphomas harbored lossof-function ARID1A mutations (p.Q482X and p.727_730del). Interestingly, the ARID1A-mutated diffuse large B-cell lymphoma sample (IE-39; p.Q1363X) also contained one of the aforementioned activating EZH2 Y646 mutations (p.Y646S) (Figure 1c). Lastly, we identified prioritized mutations in other chromatin-modifying genes including loss-of-function mutations in KMT2B (also known as MLL2 or MLL4; p.Q2495X) and KMT3B (also known as NSD1; p.Q1532X) in one case each of the follicular lymphoma and diffuse large B-cell lymphoma, respectively (Figure 1c).

All mutations in the above chromatin modeling genes in our cohort were heterozygous based on estimated tumor content and variant allele frequencies. Hence, to determine the impact of mutations on protein expression, we assessed ARID1A, EZH2 and KMT3B expression in several lymphoma samples by immunohistochemistry. Although limited by the number of mutant samples, immunohistochemical expression of ARID1A in wild-type and mutant follicular lymphoma samples showed no qualitative differences, as ARID1A protein was very low or absent in both samples (Figure 2a, positive control not shown). In contrast, the diffuse large B-cell lymphoma harboring the loss-of-function KMT3B (NSD1) p.Q1532X nonsense mutation showed decreased KMT3B expression compared with a diffuse large B-cell lymphoma sample without prioritized KMT3B alteration (Figure 2b). EZH2 expression was retained in two samples that each harbored prioritized p.Y646N or p.Y646S mutations, which are known gain-of-function mutations (Figure 2c).

In addition to MYD88 and chromatin-modifying genes, we also identified samples with prioritized alterations in well-known tumor suppressors and oncogenes (Figure 1c and Supplementary Table S2). Three diffuse large B-cell lymphomas harbored single loss-of-function mutations in phosphatase and tensin homolog (PTEN), ataxia telangiectasia mutation (ATM), or neurofibromin 1 (NF1). Of interest, sample IE-07, a follicular lymphoma, harbored biallelic tumor protein 53 (TP53) loss-offunction hotspot mutations (R248 and G244). Another follicular lymphoma contained a TP53 non-frameshift deletion (p.255-256del), while a diffuse large B-cell lymphoma harbored a TP53 R175 hotspot missense mutation. Loss-of-function nonsense mutations were also observed in the tumor suppressor split end family protein (SPEN) in one case each of diffuse large B-cell lymphoma and follicular lymphoma. Prioritized alterations in oncogenes included missense mutations in neuroblastoma rat sarcoma viral oncogene homolog (NRAS, Q61K) and Harvey rat sarcoma viral oncogene homolog (HRAS, G60S) in a single diffuse large B-cell lymphoma and follicular lymphoma sample, respectively.

Figure 2 Immunohistochemistry for chromatin-modifying proteins in mutant ARID1A, NSD1, and EZH2 lymphoma samples. Hematoxylin and eosin (H\&E, a, c and e) stains (large panels, original magnifications: $\times 400$, insets $\times 100$ ) and corresponding immunohistochemistry (b, $\mathbf{d}$ and $\mathbf{f}$, original magnifications: $\times 400)$ for indicated chromatin-modifying proteins was performed in selected orbital and ocular adnexal lymphomas based on mutational status. (a and b) IE-07, a usual appearing follicular lymphoma (a) with a lossof-function ARID1A mutation (p.Q842X), demonstrates absent ARID1A expression (b). (c and d). IE-39, a diffuse large B-cell and marginal zone lymphoma with a truncating stopgain mutation in NSD1 (p.Q1532X), shows very weak expression of NSD1 (d) in tumor cells (wildtype NSD1 diffuse large B-cell and marginal zone lymphoma samples showed robust expression of NSD1 by immunohistochemistry, see Supplementary Figure). IE-17, a follicular lymphoma (e) with a predicted gain-of-function variant in EZH2 (p.Y646N), shows robust EZH2 (f) expression (wild-type EZH2 marginal zone lymphoma samples did not show EZH2 expression by immunohistochemistry, see Supplementary Figure). 
In addition to single nucleotide alterations and short indels, we used NGS to assess copy number alterations in genes targeted by our panel using a validated approach. ${ }^{33}$ Overall, we identified relatively few focal, high-level amplifications or deletions in our orbital and ocular adnexal lymphoma cohort (Figure 1c). We identified high level, prioritized deletions in CDKN2A ( $p 16 I N K 4 A$ ) in three diffuse large B-cell lymphomas and one marginal zone lymphoma, as well as a high-level deletion in PTEN

IE-07 (FL) ARID1A p.Q482X
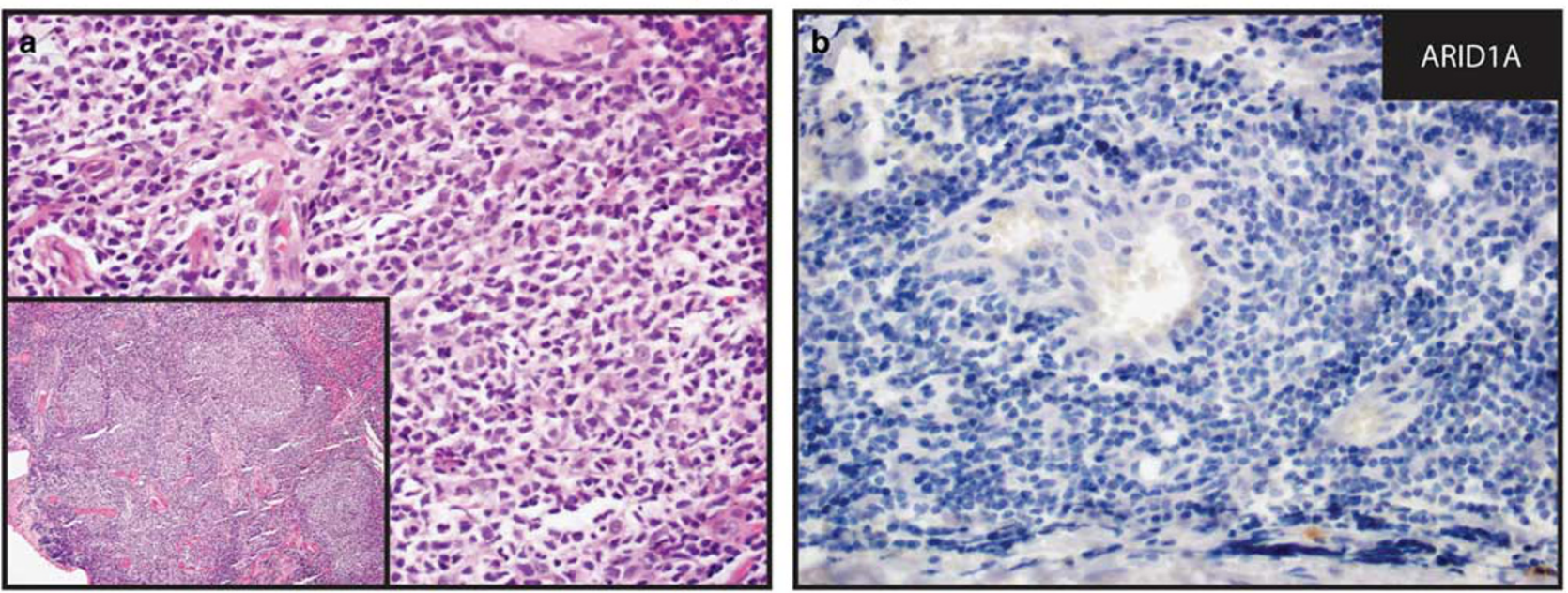

IE-39 (DLBCL) NSD1 p.Q1532X
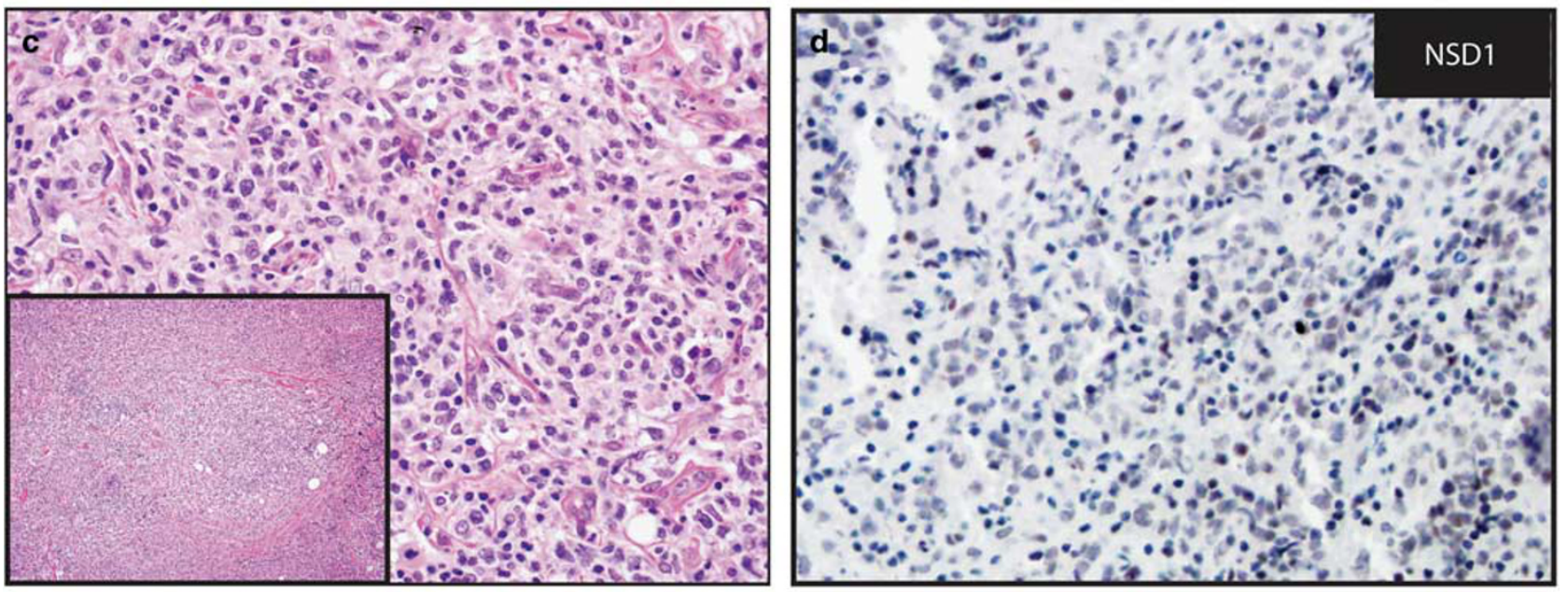

IE-17 (FL) EZH2 p.Y646N
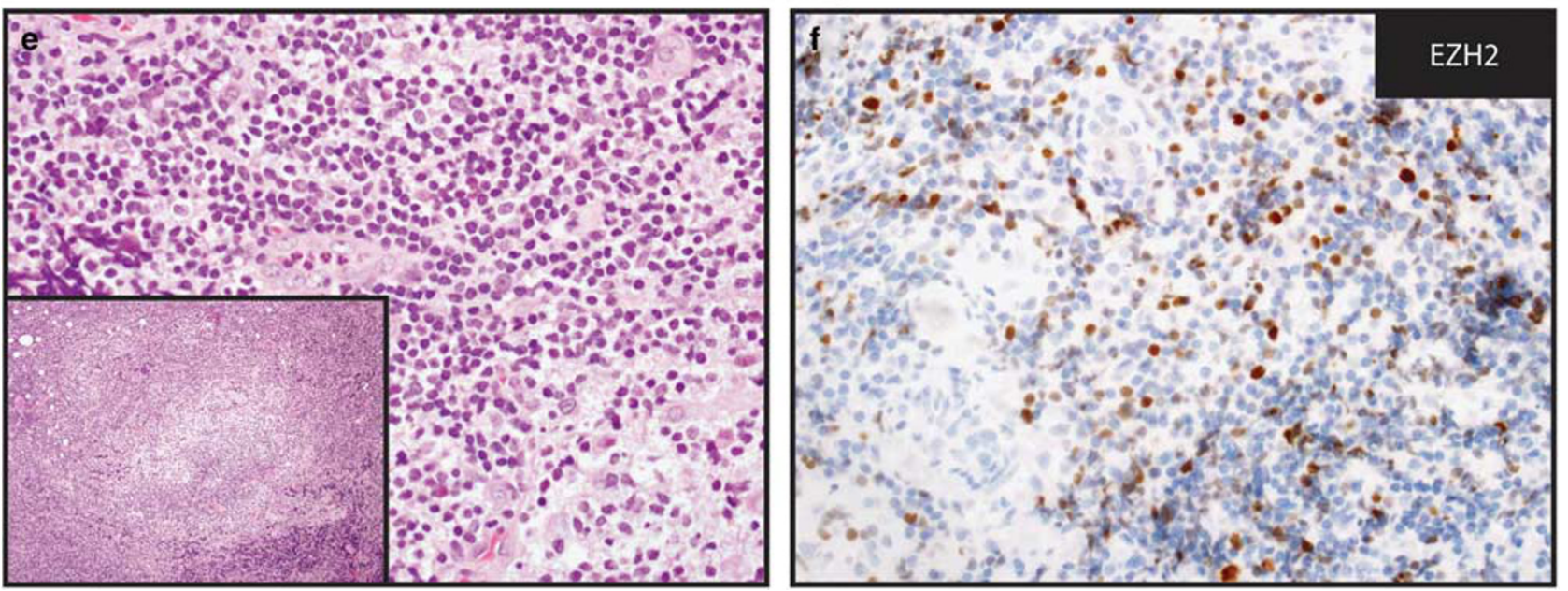
in a diffuse large B-cell lymphoma. Finally, although not prioritized, broad low-level gains/losses were observed, including one-copy gains in chromosomes 12 and 3 in a diffuse large B-cell lymphoma and a marginal zone lymphoma, respectively, as well as one-copy losses in 17p in two diffuse large B-cell lymphomas. Cohort-wide copy number plots are shown in Figure 3a, with individual amplicon level plots for one sample each for diffuse large B-cell lymphoma, follicular lymphoma, and marginal zone lymphoma (Figure 3b). Among other copy number changes, we found high-level deletions in CDKN2A and PTEN (IE-18, diffuse large B-cell lymphoma), and CDKN2A (IE-29, marginal zone lymphoma), whereas no copy number gains or losses were observed in any of the follicular lymphomas profiled (IE-17, representative plot) (Figure 3b).

\section{Discussion}

We performed targeted NGS of 38 formalin-fixed, paraffin-embedded orbital and ocular adnexal lymphomas (marginal zone, follicular, and diffuse large B-cell lymphoma subtypes), to identify somatic mutations and copy number alterations associated with tumorigenesis, and identify novel potential therapeutic strategies (Figure 4). Importantly, although numerous studies have profiled extraorbital and ocular adnexal NHLs and B-cell neoplasms using NGS, orbital and ocular adnexal lymphomas have not been profiled by comprehensive approaches, and it is unclear whether these cancers share similar alterations and potential therapeutic targets. ${ }^{38-43}$

The most frequently mutated gene in our cohort was MYD88, with $28 \%$ of orbital and ocular adnexal lymphomas harboring gain-of-function hotspot mutations. A major finding of this work was that the frequencies of $M Y D 88$ mutations in marginal zone and diffuse large B-cell lymphomas of the orbital and ocular adnexal regions was higher than those previously reported for these subtypes in locations outside the orbit and ocular adnexa. Similar MYD88 mutations are frequent in non-orbit and ocular adnexal B-cell neoplasms, including Waldenström's macroglobulinemia (79-100\%) and diffuse large B-cell lymphomas (6-39\%), but remain uncommon in marginal zone lymphomas (415\%). ${ }^{35,44-46}$ Among low-grade B-cell lymphomas, the L265P MYD88 mutation is frequently associated with lymphoplasmacytic lymphoma (of which Waldenström's macroglobulinemia is an IgM-producing subtype). ${ }^{47}$ In our cohort of MYD88 mutant marginal zone lymphoma cases, lymphoplasmacytic lymphomas were excluded in each case on morphologic, immunophenotypic, and clinical grounds. No patient demonstrated the presence of urinary or serum paraprotein, no amyloid deposition was present, and CD38 expression (dim) was observed in only one case. Although lymphoplasmacytic lymphomas have been rarely described in the orbit and ocular adnexa, nearly all of these cases have been preceded by a history of lymphoma/Waldenström's macroglobulinemia or were known to have widespread disease at the time of ocular involvement. ${ }^{48,49}$ Importantly, all of our marginal zone lymphoma cases with MYD88 mutations were isolated primary ocular tumors. Plasmacytic differentiation has been described frequently in ocular marginal zone lymphomas ${ }^{6,48,50}$ and was noted by our hematopathologists in varying degrees among our MYD88 mutant marginal zone lymphomas. Indeed, the possible correlation between plasmacytic differentiation and MYD88 mutations is an intriguing topic for future, expanded studies of marginal zone lymphomas of the orbit and ocular adnexa.

MYD88 mutations have been reported infrequently $(0-7 \%)$ in non-lymphoplasmacytic lymphomas and extranodal marginal zone lymphomas, ${ }^{41,46,47,51-53}$ the most common form (76-100\%) of orbital and ocular adnexal lymphomas. ${ }^{41,46,47,51,54}$ Hence, the observed rates of MYD88 mutations in our marginal zone lymphomas (25\%) and diffuse large B-cell lymphomas $(71 \%)$ are notably higher than those previously reported in NHLs outside the orbit and ocular adnexa. Interestingly, all MYD88 mutant diffuse large B-cell lymphomas of the orbit and ocular adnexa were of the non-germinal center B-cell (activated B-cell) subtype, consistent with previous reports that link MYD88 alterations to activated B-cell diffuse large B-cell lymphoma subtypes in extranodal NHLs outside the orbit and ocular adnexa. ${ }^{45}$

The fact that lymphomas in the orbit and ocular adnexa have different rates of somatic alterations than lymphomas outside of this region is not new. For instance, MALT1/IGH translocations are relatively rare in marginal zone lymphomas of the orbital and ocular adnexal region when compared with marginal zone lymphomas outside this location. ${ }^{55}$ The distinct frequency of genetic lesions such as MYD88 mutations and MALT1/IGH rearrangements in our cohort of diffuse large B-cell lymphomas and marginal zone lymphomas versus similar subtypes in lymphomas outside the orbit and ocular adnexa may suggest that additional mechanisms or selection events are involved during lymphomagenesis in the orbit and ocular adnexa, a unique extranodal site. In addition, as our panel did not include MALT1/IGH rearrangements, we were unable to assess whether an association with this rearrangement and MYD88 alterations exists, or whether these are mutually exclusive in diffuse large B-cell lymphomas and marginal zone lymphomas of the orbit and ocular adnexa. More research with a larger number of orbital and ocular adnexal lymphomas will be important for confirmation of these findings, assessment of whether MALT1/IGH rearrangements occur jointly or independently with MYD88 mutations, and for investigation of the potential unique roles 

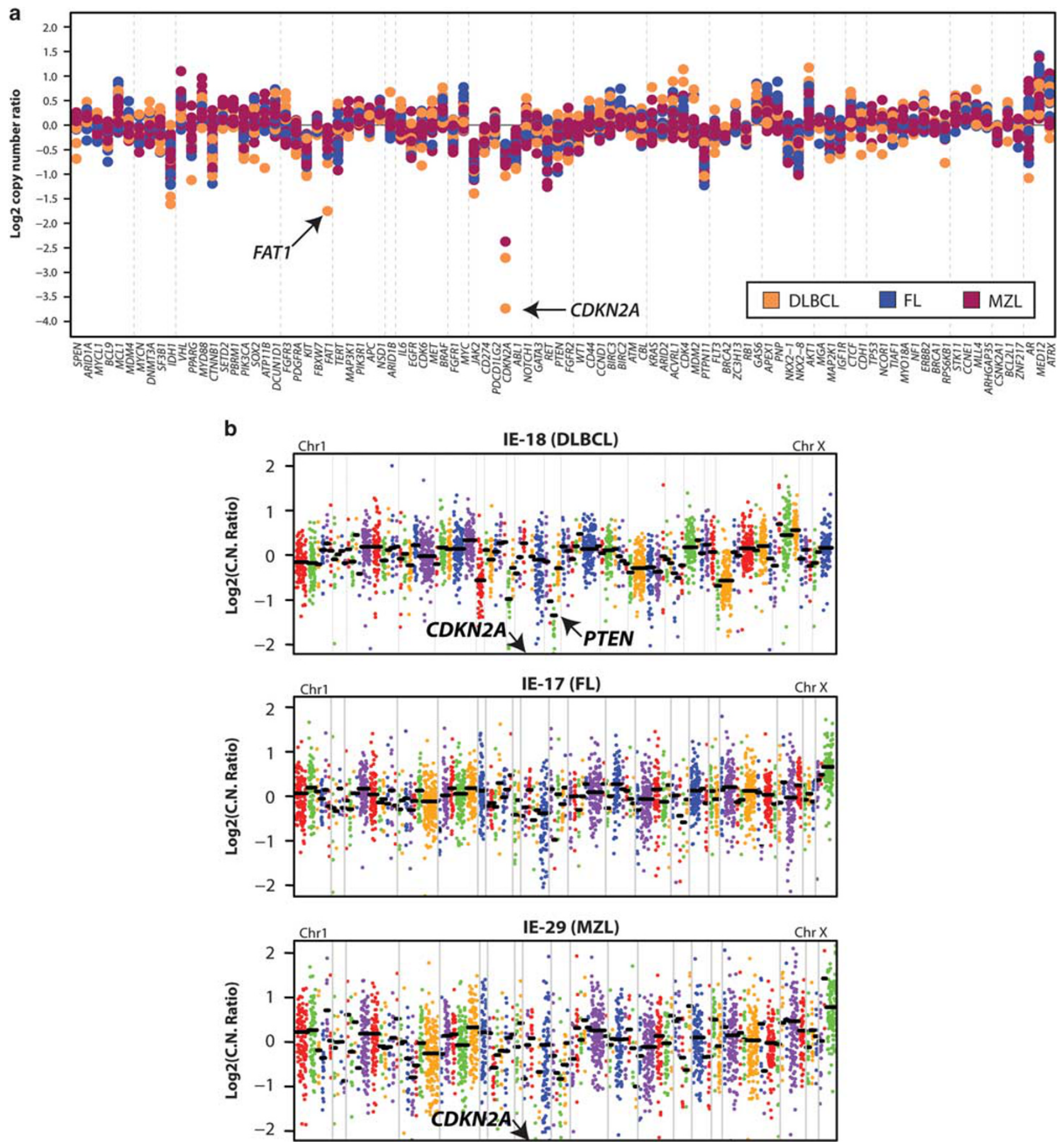

Figure 3 Copy number analysis of orbital and ocular adnexal lymphomas from next-generation sequencing (NGS) data. For each sequenced orbital and ocular adnexal lymphoma, GC-content corrected, normalized read counts per amplicon were divided by those from a composite normal sample, yielding a tumor-to-normal copy number ratio for each amplicon. Gene-level copy number estimates were determined by taking the weighted mean of the per-probe copy number ratios. (a) Summary of gene-level copy number ratios (log ${ }_{2}$ ) for all profiled orbital and ocular adnexal lymphomas stratified by subtype according to the legend. Selected genes of interest with high-level copy number alteration are indicated. Copy number plot for IE-23 was not informative and was removed from Figure 3a. (b). Copy number profiles for three individual orbital and ocular adnexal lymphomas, IE-18 (diffuse large B-cell lymphoma, DLBCL), IE-17 (follicular lymphoma, FL), and IE-29 (marginal zone lymphoma, MZL). $\log _{2}$ tumor-to-normal copy number ratios per amplicon are plotted (with each individual amplicon represented by a single dot and each individual gene indicated by different colors), with gene-level copy number estimates (black bars) determined by taking the weighted mean of the per-probe copy number ratios. Selected high-level copy number alterations are indicated. $\log _{2}$ copy number ratios for $C D K N 2 A$ in both IE-18 and IE-29 are off the scale (IE-18=-4.26; IE-29=-2.37). IE-17 shows no copy number changes. 

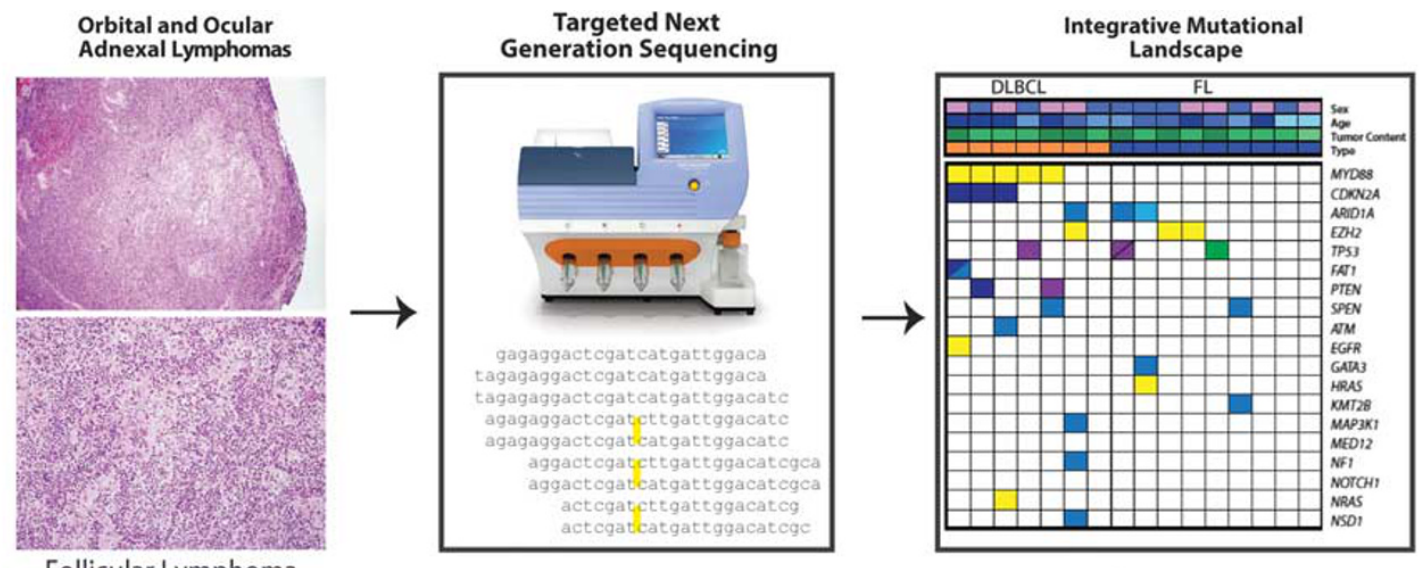

Follicular Lymphoma

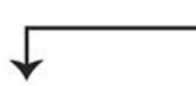

Copy Number Analysis

Potential Therapeutic Targets
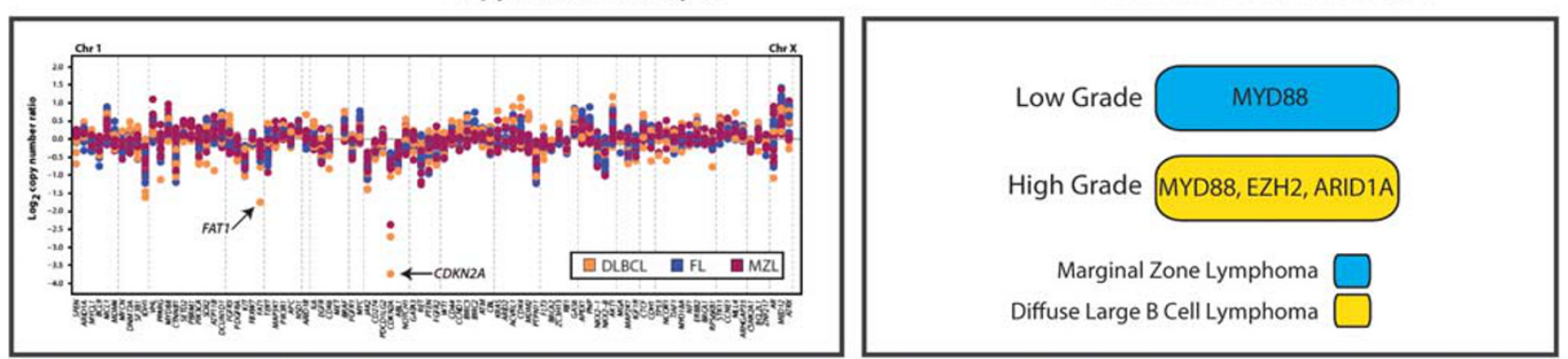

Figure 4 Workflow of determining driver and potentially actionable genomic alterations. Genomic DNA from formalin-fixed, paraffinembedded orbital and ocular lymphoma tissue enriched by macrodissection is subjected to targeted next-generation sequencing (NGS) using a cancer gene panel. Bioinformatics analysis yields candidate point mutations, small indels and copy number alterations that potentially drive tumor growth and development in orbital and ocular adnexal lymphomas. Potential therapeutic targets are prioritized and reported.

these genetic lesions play in the etiology of these tumors in this site.

Importantly, in addition to numerous clinical trials targeting downstream MYD88-dependent factors in the $\mathrm{NF}-\kappa \mathrm{B}$ signaling pathway (such as phosphorylated Bruton tyrosine kinase and interleukin-1 receptor-associated kinases 1 and 4), and those stratifying response by MYD88 p.L265P mutation status, currently recruiting clinical trials in diffuse large B-cell lymphomas assessing TLR inhibitors require the MYD88 p.L265P mutation as an entry criterion (https://clinicaltrials.gov/ct2/results?term = myd88; accessed 23 August 2015). In summary, our results support evaluation of therapeutic strategies targeting MYD88 and downstream mediators activated by gain-of-function mutations in diffuse large B-cell lymphomas and marginal zone lymphomas of the orbit and ocular adnexa.

Alterations in chromatin-modifying genes such as those that encode histone lysine methyltransferases (eg, KMT2B, KMT3B, and EZH2) and ATP-dependent chromatin remodelers (eg, ARID1A) are among the most common mutations in human cancers, including many forms of B-cell lymphomas, especially diffuse large B-cell lymphomas and follicular lymphomas. ${ }^{7,36-39,56-61}$ Indeed, in our cohort, priori- tized mutations in chromatin-modifying genes were found only in diffuse large B-cell lymphomas and follicular lymphomas (6 of 16 (38\%) with prioritized alterations). Specifically, 2 of $9(22 \%)$ follicular lymphomas and 1 of 7 (14\%) diffuse large B-cell lymphomas harbored prioritized EZH2 or ARID1A mutations, rates that are consistent with extra-orbital and ocular adnexal diffuse large B-cell lymphomas and follicular lymphomas. ${ }^{36-38,40}$ The single EZH2 mutant diffuse large B-cell lymphoma case in our cohort was of the germinal center B-cell phenotype, consistent with NHLs outside the orbit and ocular adnexa. ${ }^{38}$ Given its association with poor prognosis in several cancers, there has been intense interest in the development of EZH2 inhibitors. ${ }^{62-64}$ At present, there are three trials (https://clinicaltrials.gov/ct2/ results?term = ezh2; accessed 23 August 2015) currently recruiting individuals with relapsed or refractory B-cell lymphomas (including diffuse large B-cell lymphomas and follicular lymphomas) for evaluation of oral EZH2 inhibitors in phase 1 and 2 clinical trials. In these trials, the presence of gain-of-function EZH2 mutations will be tested, in order to determine in which arm an affected individual will enroll (wild type or mutant EZH2 status). Likewise, a recent report demonstrated that loss-of-function ARID1A 
mutations in ovarian clear cell carcinomas render these tumors sensitive to EZH2 inhibitors in vitro and in vivo, ${ }^{65}$ suggesting additional ways to target alterations in histone modifiers based on synthetic lethality. Of interest, a diffuse large B-cell lymphoma sample (IE-39) contained both a gain-offunction EZH2 mutation (p.Y646S) and a loss-offunction ARID1A mutation (p.Q1363X), suggesting potentially remarkable sensitivity to EZH2 inhibition. In summary, our results support frequent potentially targetable alterations in histone/chromatin modifiers in diffuse large B-cell lymphomas and follicular lymphomas of the orbit and ocular adnexa, consistent with results in non-orbital and ocular adnexal NHLs.

In recent years, significant interest has emerged in the potential clinical value of NGS of tumoral DNA, in order to discover or guide strategies that link personalized therapies to specific genomic alterations present in the cancer. For the first time, we apply comprehensive next-generation genomic profiling to low- and high-grade formalin-fixed, paraffinembedded orbital and ocular adnexal lymphomas, cancer subtypes for which comprehensive NGSbased profiling has not been reported. Of note, the NGS panel used herein was designed to target pancancer (including lymphoma) altered genes filtered to those with near-term potential actionability and a modified, lymphoma/solid tumor-specific version ${ }^{26}$ is being used in the NCI-MATCH trial, a multi-site basket trial sponsored by the National Cancer Institute, which aims to match patients to investigational therapeutics based on their prioritized mutation profile rather than the site of tumor origin. We did not analyze non-tumor cells from the corresponding orbital and ocular adnexal lymphoma samples; thus, to distinguish germline from somatic variants, we cross-referenced candidate variants with data from the NHLBI Grand Opportunity Exome Sequencing Project (ESP6500) and the 1000 Genomes project, which include normal variants from several thousand individuals, and took into account variant fractions (for details, see Materials and Methods). Taken together, we have previously confirmed that our filtering criteria (Materials and Methods) identify prioritized high-confidence somatic variants from formalin-fixed, paraffinembedded tissue, which pass Sanger sequencing validation with $>95 \%$ accuracy, which is a nearly identical process as that used in the NCI-MATCH Trial, which also does not analyze non-tumor tissue from the corresponding lymphoma or solid tumor samples. ${ }^{26,28-32}$

Limitations of our study include a single site cohort, relatively few samples for each subtype, and the use of a targeted panel-based approach without assessment of other key alterations (eg, chromosomal rearrangements, methylation, and transcriptional profiling). Hence, larger, prospective, multi-institutional studies will be required to confirm our findings and clinical trials are needed in orbital and ocular adnexal lymphomas to test potential treatment strategies proposed herein.

In conclusion, through NGS targeting informative/ potentially actionable genomic alterations, we identified recurrent mutations and copy number alterations across 36 orbital and ocular adnexal lymphomas, including diffuse large B-cell, follicular, and marginal zone lymphoma subtypes. There are few, if any reports, which have comprehensively profiled primary orbital and ocular adnexal lymphomas, especially diffuse large B-cell and follicular lymphomas, which is a unique feature of this study. We identified MYD88 gain-of-function mutations at higher rates in orbital/ocular adnexal diffuse large B-cell lymphomas and follicular lymphomas $(71 \%$ and $25 \%$, respectively) than those reported in nonorbital/ocular sites; nearly all of these tumors were primary to the orbit or ocular adnexa (Figure 1c). Likewise, histone/chromatin modifiers showed frequent alterations in our cohort (at similar rates to non-orbital/ocular adnexal sites), including potentially targetable gain-of-function mutations in the histone methyltransferase EZH2 among diffuse large B-cell lymphomas and follicular lymphomas of the orbit and ocular adnexa.

Importantly, $25 \%$ of the sequenced samples carried specific GoF mutations in MYD88 (diffuse large B-cell and marginal zone lymphomas) and EZH2 (diffuse large B-cell and follicular lymphomas). These specific alterations satisfy entry criteria for four ongoing clinical trials: three that use EZH2 pharmacologic inhibitors (NCT01897571, NCT02082977, and NCT02395601) in any type of B-cell lymphoma and one that uses an oligonucleotide (IMO-8400) to inhibit the activity of oncogenic L265P mutant MYD88 protein (NCT02252146) in diffuse large B-cell lymphomas. As MYD88 mutations are even more common among diffuse large B-cell and marginal zone lymphomas of the orbit and ocular adnexa than similar subtypes outside of this region, therapies targeting this lesion may be even more effective for diffuse large B-cell and marginal zone lymphomas occurring in these locations. As novel therapeutic approaches are urgently needed for orbital and ocular adnexal lymphomas, owing to poor survival rates and high rates of relapse (diffuse large B-cell lymphomas), as well as local and visionrelated toxicities of present treatments (follicular and marginal zone lymphomas), our results demonstrate the utility of a next-generation-based approach to nominate precision therapeutic approaches for orbital and ocular adnexal lymphomas, and other intraocular, ocular adnexal, and orbital tumors.

\section{Acknowledgments}

This work was supported by the National Eye Institute (K12EY022299), National Cancer InstituteUniversity of Michigan Comprehensive Cancer Center Support Grant (P30CA046592), The Leonard 
G. Miller Ophthalmic Research Fund at the Kellogg Eye Center, Barbara Dunn Research Fund, Beatrice \& Reymont Paul Foundation and March Hoops to Beat Blindness to RCR. RCR is the Leslie H. and Abigail S. Wexner Emerging Scholar and SAT is the A. Alfred Taubman Emerging Scholar, both of the A. Alfred Taubman Medical Research Institute, which supported, in part, this study.

\section{Disclosure/conflict of interest}

SAT has received travel support and had a separate sponsored research agreement with Compendia Bioscience/Life Technologies/ThermoFisher Scientific, which provides access to the sequencing panel used herein. ${ }^{26}$ No other aspect of the study described herein was supported by Compendia Bioscience/Life Technologies/ThermoFisher Scientific. All other authors declare no conflicts of interest.

\section{References}

1 Margo CE, Mulla ZD. Malignant tumors of the orbit. analysis of the Florida Cancer Registry. Ophthalmology 1998;105:185-190.

2 Spraul CW, Grossniklaus HE. Analysis of 24,444 surgical specimens accessioned over 55 years in an ophthalmic pathology laboratory. Int Ophthalmol 1997; 21:283-304.

3 Moslehi R, Devesa SS, Schairer C et al. Rapidly increasing incidence of ocular non-Hodgkin lymphoma. J Natl Cancer Inst 2006;98:936-939.

4 Fung CY, Tarbell NJ, Lucarelli MJ et al. Ocular adnexal lymphoma: clinical behavior of distinct World Health Organization classification subtypes. Int J Radiat Oncol Biol Phys 2003;57:1382-1391.

5 White WL, Ferry JA, Harris NL et al. Ocular adnexal lymphoma. A clinicopathologic study with identification of lymphomas of mucosa-associated lymphoid tissue type. Ophthalmology 1995;102:1994-2006.

6 Stefanovic A, Lossos IS. Extranodal marginal zone lymphoma of the ocular adnexa. Blood 2009;114: 501-510.

7 Pasqualucci L, Trifonov V, Fabbri G et al. Analysis of the coding genome of diffuse large B-cell lymphoma. Nat Genet 2011;43:830-837.

8 Lohr JG, Stojanov P, Lawrence MS et al. Discovery and prioritization of somatic mutations in diffuse large B-cell lymphoma (DLBCL) by whole-exome sequencing. Proc Natl Acad Sci USA 2012;109:3879-3884.

9 Tsujimoto Y, Cossman J, Jaffe E et al. Involvement of the bcl-2 gene in human follicular lymphoma. Science 1985;228:1440-1443.

10 Limpens J, de Jong D, van Krieken JH et al. Bcl-2/JH rearrangements in benign lymphoid tissues with follicular hyperplasia. Oncogene 1991;6:2271-2276.

11 Okosun J, Bodor C, Wang J et al. Integrated genomic analysis identifies recurrent mutations and evolution patterns driving the initiation and progression of follicular lymphoma. Nat Genet 2014;46:176-181.

12 Oricchio E, Nanjangud G, Wolfe AL et al. The Ephreceptor A7 is a soluble tumor suppressor for follicular lymphoma. Cell 2011;147:554-564.
13 Zhu D, McCarthy H, Ottensmeier CH et al. Acquisition of potential N-glycosylation sites in the immunoglobulin variable region by somatic mutation is a distinctive feature of follicular lymphoma. Blood 2002;99:2562-2568.

14 De Cicco L, Cella L, Liuzzi R et al. Radiation therapy in primary orbital lymphoma: a single institution retrospective analysis. Radiat Oncol 2009;4:60.

15 Esik O, Ikeda H, Mukai K et al. A retrospective analysis of different modalities for treatment of primary orbital non-Hodgkin's lymphomas. Radiother Oncol 1996;38: 13-18.

16 Stafford SL, Kozelsky TF, Garrity JA et al. Orbital lymphoma: radiotherapy outcome and complications. Radiother Oncol 2001;59:139-144.

17 Kaushik M, Pulido JS, Schild SE et al. Risk of radiation retinopathy in patients with orbital and ocular lymphoma. Int J Radiat Oncol Biol Phys 2012;84: 1145-1150.

18 Suh CO, Shim SJ, Lee SW et al. Orbital marginal zone B-cell lymphoma of MALT: radiotherapy results and clinical behavior. Int J Radiat Oncol Biol Phys 2006;65: 228-233.

19 Rasmussen PK, Coupland SE, Finger PT et al. Ocular adnexal follicular lymphoma: a multicenter international study. JAMA Ophthalmol 2014;132:851-858.

20 Munch-Petersen HD, Rasmussen PK, Coupland SE et al. Ocular adnexal diffuse large B-cell lymphoma: a multicenter international study. JAMA Ophthalmol 2015;133:165-173.

21 Kim WS, Honma K, Karnan S et al. Genome-wide arraybased comparative genomic hybridization of ocular marginal zone B cell lymphoma: comparison with pulmonary and nodal marginal zone B cell lymphoma. Genes Chromosomes Cancer 2007;46:776-783.

22 Matteucci C, Galieni P, Leoncini L et al. Typical genomic imbalances in primary MALT lymphoma of the orbit. J Pathol 2003;200:656-660.

23 Ruiz A, Reischl U, Swerdlow SH et al. Extranodal marginal zone B-cell lymphomas of the ocular adnexa: multiparameter analysis of 34 cases including interphase molecular cytogenetics and PCR for Chlamydia psittaci. Am J Surg Pathol 2007;31:792-802.

24 Schiby G, Polak-Charcon S, Mardoukh C et al. Orbital marginal zone lymphomas: an immunohistochemical, polymerase chain reaction, and fluorescence in situ hybridization study. Hum Pathol 2007;38: 435-442.

25 Tanimoto K, Sekiguchi N, Yokota Y et al. Fluorescence in situ hybridization (FISH) analysis of primary ocular adnexal MALT lymphoma. BMC cancer 2006; $6: 249$.

26 Hovelson DH, McDaniel AS, Cani AK et al. Development and validation of a scalable next-generation sequencing system for assessing relevant somatic variants in solid tumors. Neoplasia 2015;17:385-399.

27 Conley BA, Doroshow JH. Molecular analysis for therapy choice: NCI MATCH. Semin Oncol 2014;41: 297-299.

28 Cani AK, Hovelson DH, McDaniel AS et al. Next-gen sequencing exposes frequent MED12 mutations and actionable therapeutic targets in phyllodes tumors. Mol Cancer Res 2015;13:613-619.

29 Warrick JI, Hovelson DH, Amin A et al. Tumor evolution and progression in multifocal and paired non-invasive/invasive urothelial carcinoma. Virchows Arch 2015;466:297-311. 
30 McDaniel AS, Stall JN, Hovelson DH et al. Nextgeneration sequencing of tubal intraepithelial carcinomas. JAMA Oncol 2015;1:1128-1132.

31 McDaniel AS, Hovelson DH, Cani AK et al. Genomic profiling of penile squamous cell carcinoma reveals new opportunities for targeted therapy. Cancer Res 2015;75:5219-5227.

32 McDaniel AS, Zhai Y, Cho KR et al. HRAS mutations are frequent in inverted urothelial neoplasms. Hum Pathol 2014;45:1957-1965.

33 Grasso C, Butler T, Rhodes $\mathrm{K}$ et al. Assessing copy number alterations in targeted, amplicon-based nextgeneration sequencing data. J Mol Diagn 2015;17:53-63.

34 Hans CP, Weisenburger DD, Greiner TC et al. Confirmation of the molecular classification of diffuse large B-cell lymphoma by immunohistochemistry using a tissue microarray. Blood 2004;103:275-282.

35 Wang JQ, Jeelall YS, Ferguson LL et al. Toll-like receptors and cancer: MYD88 mutation and inflammation. Front Immunol 2014;5:367.

36 Bodor C, Grossmann V, Popov N et al. EZH2 mutations are frequent and represent an early event in follicular lymphoma. Blood 2013;122:3165-3168.

37 Bodor C, O'Riain C, Wrench D et al. EZH2 Y641 mutations in follicular lymphoma. Leukemia 2011;25:726-729.

38 Morin RD, Johnson NA, Severson TM et al. Somatic mutations altering EZH2 (Tyr641) in follicular and diffuse large B-cell lymphomas of germinal-center origin. Nat Genet 2010;42:181-185.

39 Morin RD, Mendez-Lago M, Mungall AJ et al. Frequent mutation of histone-modifying genes in non-Hodgkin lymphoma. Nature 2011;476:298-303.

$40 \mathrm{Li} \mathrm{H}$, Kaminski MS, Li Y et al. Mutations in linker histone genes HIST1H1 B, C, D and E, OCT2 (POU2F2), IRF8 and ARID1A underlying the pathogenesis of follicular lymphoma. Blood 2014;123:1487-1498.

$41 \mathrm{Li}$ ZM, Rinaldi A, Cavalli A et al. MYD88 somatic mutations in MALT lymphomas. Br J Haematol 2012; 158:662-664.

42 Tierens A, Delabie J, Pittaluga S et al. Mutation analysis of the rearranged immunoglobulin heavy chain genes of marginal zone cell lymphomas indicates an origin from different marginal zone B lymphocyte subsets. Blood 1998;91:2381-2386.

43 Streubel B, Simonitsch-Klupp I, Mullauer L et al. Variable frequencies of MALT lymphoma-associated genetic aberrations in MALT lymphomas of different sites. Leukemia 2004;18:1722-1726.

44 Treon SP, Xu L, Yang G et al. MYD88 L265P somatic mutation in Waldenstrom's macroglobulinemia. N Engl J Med 2012;367:826-833.

45 Ngo VN, Young RM, Schmitz R et al. Oncogenically active MYD88 mutations in human lymphoma. Nature 2011;470:115-119.

46 Martinez-Lopez A, Curiel-Olmo S, Mollejo M et al. MYD88 (L265P) somatic mutation in marginal zone B-cell lymphoma. Am J Surg Pathol 2015;39:644-651.

47 Gachard N, Parrens M, Soubeyran I et al. IGHV gene features and MYD88 L265P mutation separate the three marginal zone lymphoma entities and Waldenstrom macroglobulinemia/lymphoplasmacytic lymphomas. Leukemia 2013;27:183-189.
48 Ferry JA, Fung CY, Zukerberg L et al. Lymphoma of the ocular adnexa: a study of 353 cases. Am J Surg Pathol 2007;31:170-184.

49 McKelvie PA. Ocular adnexal lymphomas: a review. Adv Anat Pathol 2010;17:251-261.

50 Lee MJ, Kim N, Choe JY et al. Clinicopathological analysis of ocular adnexal extranodal marginal Zone B-cell lymphoma with IgG4-positive cells. PLoS ONE 2015;10:e0131458.

51 Zhu D, Ikpatt OF, Dubovy SR et al. Molecular and genomic aberrations in Chlamydophila psittaci negative ocular adnexal marginal zone lymphomas. Am J Hematol 2013;88:730-735.

52 Liu F, Karube K, Kato $\mathrm{H}$ et al. Mutation analysis of NF-kappaB signal pathway-related genes in ocular MALT lymphoma. Int J Clin Exp Pathol 2012;5: 436-441.

53 Martinez-Climent JA. The origin and targeting of mucosa-associated lymphoid tissue lymphomas. Curr Opin Hematol 2014;21:309-319.

54 Lee JL, Kim MK, Lee KH et al. Extranodal marginal zone B-cell lymphomas of mucosa-associated lymphoid tissue-type of the orbit and ocular adnexa. Ann Hematol 2005;84:13-18.

55 Sjo LD. Ophthalmic lymphoma: epidemiology and pathogenesis. Acta Ophthalmol 2009;87 Thesis 1: $1-20$.

56 Rao RC, Dou Y. Hijacked in cancer: the KMT2 (MLL) family of methyltransferases. Nat Rev Cancer 2015;15: 334-346.

57 Kandoth C, McLellan MD, Vandin F et al. Mutational landscape and significance across 12 major cancer types. Nature 2013;502:333-339.

58 Smedby KE, Foo JN, Skibola CF et al. GWAS of follicular lymphoma reveals allelic heterogeneity at 6 p21.32 and suggests shared genetic susceptibility with diffuse large B-cell lymphoma. PLoS Genet 2011;7: e1001378.

$59 \mathrm{Li} \mathrm{H}$, Kaminski MS, Li Y et al. Mutations in linker histone genes HIST1H1 B, C, D, and E; OCT2 (POU2F2); IRF8; and ARID1A underlying the pathogenesis of follicular lymphoma. Blood 2014;123:1487-1498.

60 Love C, Sun Z, Jima D et al. The genetic landscape of mutations in Burkitt lymphoma. Nat Genet 2012;44: 1321-1325.

61 Zhang J, Grubor V, Love CL et al. Genetic heterogeneity of diffuse large B-cell lymphoma. Proc Natl Acad Sci USA 2013;110:1398-1403.

62 McCabe MT, Ott HM, Ganji G et al. EZH2 inhibition as a therapeutic strategy for lymphoma with EZH2activating mutations. Nature 2012;492:108-112.

63 Knutson SK, Wigle TJ, Warholic NM et al. A selective inhibitor of EZH2 blocks H3K27 methylation and kills mutant lymphoma cells. Nat Chem Biol 2012;8: 890-896.

64 Bradley WD, Arora S, Busby J et al. EZH2 inhibitor efficacy in non-Hodgkin's lymphoma does not require suppression of H3K27 monomethylation. Chem Biol 2014;21:1463-1475.

65 Bitler BG, Aird KM, Garipov A et al. Synthetic lethality by targeting EZH2 methyltransferase activity in ARID1A-mutated cancers. Nat Med 2015;21:231-238.

Supplementary Information accompanies the paper on Modern Pathology website (http://www.nature.com/ modpathol) 